\title{
Exact solutions for two-dimensional nonlinear force-free magnetic fields
}

\author{
A. H. Khater ${ }^{1,2}$, S. M. Moawad ${ }^{1}$ and D. K. Callebaut ${ }^{2}$ \\ ${ }^{1}$ Department of Mathematics, Faculty of Science, Beni-Suef University, Beni-Suef, Egypt \\ email: khater_ah@hotmail.com \& smmoawad@hotmail.com \\ ${ }^{2}$ Departement Natuurkunde, CGB, University of Antwerp (UA), B-2020 Antwerp, Belgium \\ email: dirk.callebaut@ua.ac.be
}

\begin{abstract}
This article is a study of a family of nonlinear force-free magnetic fields (FFMFs), in Cartesian geometry under assumption of translational symmetry, as simple models of the magnetic fields in the solar corona. For this configuration all the physical quantities are invariant under translations in a fixed direction to be the direction $\mathrm{Oz}$ of a Cartesian coordinate system. Two classes of exact analytic solutions for the steady state are obtained. These solutions may be helpful in understanding the physics involved in the transition from the low-confinement to the high-confinement mode in tokamaks. In particular, they can be employed for stability investigations, which would be of relevance to magnetic confinement systems. Further, the obtained solutions may have several applications in the study of solar photosphere, the solar corona, as well as astrophysical plasmas.
\end{abstract}

Keywords. Exact solutions, Force-free magnetic fields, Solar corona, Solar photosphere

\section{Introduction}

The magnetic fields in the solar corona above active regions of sunspots are sufficiently strong that the magnetic field energy density greatly exceeds the plasma energy density (the plasma beta is much less than unity). The magnetic field may then be modeled as force-free. With the advent of projects aiming to the magnetic confinement of controlled thermonuclear plasma to produce fusion energy, the FFMFs have been used extensively in spheromaks and reversed field pinches. On the other hand, the magnetic clouds ejected from the Sun, producing the major perturbations to the Earth's radiation belts during the satellite era seem to possess FFMFs which have budded from the solar magnetic field. Much work has been done on magnetic field models that are translationally symmetric, since many solar active regions are observed to have arcade-like geometries (see for example Voslamber \& Callebaut (1962); Zaghloul \& Barajas (1990) ; Porter, Klimchuk \& Sturrock (1992); Low (1993); Macleod (1995); Bogoyavlenskij (2000); Gonzalez-Gascon \& Peralta-Salas (2001); Wiegelmann \& Neukirch (2003); Khater \& Moawad (2005); and references there in).

In this article, we present a simple mathematical solution describing two-dimensional FFMFs in Cartesian geometry. This solution has several interesting applications in the study of solar magnetic fields, which will be consider in section 2. For an arcade-like magnetic structure, overlying a long, relatively straight polarity-inversion line on the photosphere, we may neglect variations in the direction of that line. We take this direction to be the x-axis in standard Cartesian coordinate system with unit vectors $\mathbf{e}_{x}, \mathbf{e}_{y}$, and $\mathbf{e}_{z}$. 


\section{Exact solutions}

FFMFs are characterized by the equations

$$
\nabla \wedge \mathbf{B}=\lambda \mathbf{B}, \quad \mathbf{B} \cdot \nabla \lambda=0,
$$

where $\mathbf{B}$ is the magnetic field which satisfies the Maxwell's equation $\nabla \cdot \mathbf{B}=0$, and $\lambda$ is, in general, a function of space.

From the symmetry assumption, the divergence-free field $\mathbf{B}$ can be expressed as $\mathbf{B}=$ $\nabla \psi \wedge \mathbf{e}_{z}+B_{z} \mathbf{e}_{z}$, where $\psi$ is the poloidal magnetic flux. The second part of (2.1) then requires $\lambda$ to be a function of $\psi$ and (2.1) implies

$$
\nabla^{2} \psi+B_{z} B_{z}^{\prime}=0
$$

with $\lambda=B_{z}^{\prime}$. The prime denotes differentiation with respect to $\psi$.

When the function $\lambda$ [in (2.1)] is assumed to be constant, then (2.1), consequently, (2.2) is linear equation. The assumption of $\lambda$ being a constant is not well suited to fields produced by a finite source, such as a pair of sunspots of opposite polarity. The problem involving a variable $\lambda$ is nonlinear, so in the following we obtain exact solutions to nonlinear form of the partial differential equation (2.2) with the choice $B_{z} B_{z}^{\prime}=\alpha\left(\psi^{2}-\beta\right)$, where $\alpha$ and $\beta$ are real constants. Then (2.2) takes the form

$$
\frac{\partial^{2} \psi}{\partial x^{2}}+\frac{\partial^{2} \psi}{\partial y^{2}}+\alpha\left(\psi^{2}-\beta\right)=0
$$

We look for solutions $\psi(x, y)=\psi(\xi), \xi=x+n y$ ( $n$ is a real constant) of (2.3). Then (2.3) reduces to the following nonlinear ordinary differential equation

$$
\nu^{2} \frac{d^{2} \psi}{d \xi^{2}}+\alpha\left(\psi^{2}-\beta\right)=0, \quad \nu=n^{2}+1 .
$$

We use the tanh method which was constructed by Malfliet (1991, 1992, 1993, 1994), Malfliet \& Hereman (1996) and Fan \& Zhang (1998) to obtain exact solutions for (2.4). For this, we express the solution $\psi(\xi)$ in the form $\psi(\xi)=\sum_{i=1}^{m} a_{i} \Psi^{i}(\xi)$, where $\Psi=$ $\tanh (k \xi)$. Thus, we may propose $\psi=a_{0}+a_{1} \Psi+a_{2} \Psi^{2}$ and by substituting $\psi$ into (2.4) and equating the coefficients of like powers to zero, we get the following set of algebraic equations:

$$
\begin{gathered}
2 \nu k^{2} a_{2}+\alpha a_{0}^{2}-\alpha \beta=0, \quad-2 \nu k^{2} a_{1}+2 \alpha a_{0} a_{1}=0, \quad-8 \nu k^{2} a_{2}+\alpha\left(a_{1}^{2}+2 a_{0} a_{2}\right)=0, \\
2 \nu k^{2} a_{1}+2 \alpha a_{1} a_{2}=0, \quad 6 \nu k^{2} a_{2}+\beta a_{2}^{2}=0 .
\end{gathered}
$$

The constant $a_{1}$ must be zero in order that these equations have real solutions. Then these equations are reduced to

$$
2 \nu k^{2} a_{2}+\alpha a_{0}^{2}-\alpha \beta=0, \quad 4 \nu k^{2}-\alpha a_{0}=0, \quad 6 \nu k^{2}+\alpha a_{2}=0,
$$

which have the following sets of solutions:

$$
a_{0}= \pm 2 \sqrt{\beta}, \quad a_{2}=\mp 3 \sqrt{\beta}, \quad \nu= \pm \frac{\alpha \sqrt{\beta}}{2 k^{2}}, \quad \beta>0,
$$

From (2.5), we obtain the following classes of exact solutions to (2.3):

$$
\psi(x, y)=\sqrt{\beta}\left[2-3 \tanh ^{2}\left(k x \pm \sqrt{\mu_{1}} y\right)\right], \quad \mu_{1}=\alpha \sqrt{\beta} / 2-k^{2}, \quad \alpha \sqrt{\beta}>2 k^{2},
$$

with the magnetic field

$$
\mathbf{B}=6 \sqrt{\beta} \operatorname{sech}^{2}\left(k x \pm \sqrt{\mu_{1}} y\right) \tanh \left(k x \pm \sqrt{\mu_{1}} y\right)\left(\mp \sqrt{\mu_{1}} \mathbf{e}_{x}+k \mathbf{e}_{y}\right)+B_{z} \mathbf{e}_{z},
$$



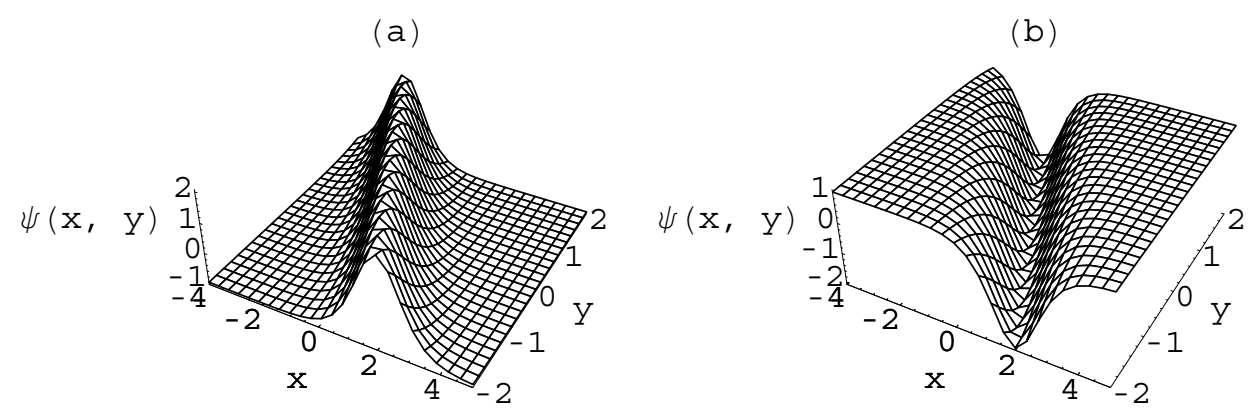

Figure 1. Soliton-like and antisoliton-like configurations for solutions (2.6) and (2.8). The values for parameters used are $\alpha=4, \beta=1$ and $k=1$ in figure 1 (a); and $\alpha=-4, \beta=1$ and $k=1$ in figure $1(b)$.

and

$$
\psi(x, y)=-\sqrt{\beta}\left[2-3 \tanh ^{2}\left(k x \pm \sqrt{\mu_{2}} y\right)\right], \quad \mu_{2}=-\left(\alpha \sqrt{\beta} / 2+k^{2}\right), \alpha \sqrt{\beta}<-2 k^{2},
$$

with the magnetic field

$$
\mathbf{B}=-6 \sqrt{\beta} \operatorname{sech}^{2}\left(k x \pm \sqrt{\mu_{2}} y\right) \tanh \left(k x \pm \sqrt{\mu_{2}} y\right)\left(\mp \sqrt{\mu_{2}} \mathbf{e}_{x}+k \mathbf{e}_{y}\right)+B_{z} \mathbf{e}_{z},
$$

The z-component of the magnetic field in (2.7) and (2.9) is determined as $B_{z}= \pm \sqrt{B_{0}+2 \alpha\left(\psi^{3} / 3-\beta \psi\right)}$, where $\psi$ is given by $(2.6)$ for $B_{z}$ in (2.7) and it is given by (2.8) for $B_{z}$ in (2.9). The constant $B_{0}$ is the value of $B_{z}^{2}$ at $\psi=0$.

Figures 1(a) and 1(b) with values of parameters listed in their captions show soliton-like and antisoliton-like configurations for the magnetic flux function described by solutions (2.6) and (2.8), respectively, in which the solutions propagate oppositely to each other.

The behavior of solutions (2.6)-(2.9) can be investigated at infinity. For solution (2.6), if $\xi \equiv k x \pm \sqrt{\mu_{1}} y \longrightarrow \pm \infty$, then $\psi \longrightarrow-\sqrt{\beta}$ and $B \longrightarrow \pm \sqrt{B_{0}+4 \alpha \beta \sqrt{\beta} / 3}$. For solution (2.8), if $\xi \equiv k x \pm \sqrt{\mu_{2}} y \longrightarrow \pm \infty$, then $\psi \longrightarrow \sqrt{\beta}$ and $B \longrightarrow \pm \sqrt{B_{0}-4 \alpha \beta \sqrt{\beta} / 3}$. Therefore all the solutions (2.6)-(2.9) are bounded at infinity, in which they are physically acceptable and have applicability in astrophysics as well as solar magnetics fields.

\section{Summary}

We have obtained two classes of exact analytic solutions for nonlinear FFMFs. The analytic solutions which we have constructed in the present work may be help in understanding the physics involved in the transition from the low-confinement to the highconfinement mode in tokamaks. Further, the obtained solutions can have several applications in the study of solar magnetic fields. A variable $\lambda$ FFMFs arises naturally in solar magnetic fields. Exact solutions were obtained previously by Biskamp (1997), Browning (1988) and Freidberg (1987) for FFMFs, that either have singularities or unboundedly grow at infinity. Such solutions have a limited applicability in astrophysics. Our obtained solutions are bounded at infinity, and haven't any singularities. 


\section{References}

Voslamber, D. \& Callebaut, D. K. 1962 Phys. Rev. 128, 2016

Zaghloul, H. \& Barajas, O. 1990 Am. J. Phys. 58, 783

Porter, L.J., Klimchuk, J.A. \& Sturrock, P.A. 1992 ApJ 385, 738

Low, B.C. 1993 ApJ 409, 798

Macleod, M. 1995 J. Math. Phys. 36, 2951; 1995 Invers Proplems 11, 1087

Bogoyavlenskij, O.I. 2000 Phys. Rev. Lett. 84, 1914

Gonzalez-Gascon, F. \& Peralta-Salas, D. 2001 Phys. Lett. A 29275

Wiegelmann, T. \& Neukirch, T. 2003 Nonlinear Proc. Geophys. 10, 313

Khater, A.H. \& Moawad, S.M. 2005 Phys. Plasmas, 12, 052902

Malfliet, W. 1991 J. Phys. A: Math. Gen. 24, 5499

Malfliet, W. 1992 Am. J. Phys. 60, 650

Malfliet, W. 1993 J. Phys. A: Math. Gen. 26, L723

Malfliet, W. 1994 De Tanh Methode in de Theorie van Niet-lineaire Golven (Thesis, Habil, Universiteit Antwerpen).

Malfliet, W. \& Hereman, W. 1996 Phys. Scripta 54, 563

Fan, E. \& Zhang, H. 1998 Phys. Lett. A 246, 403

Biskamp, D. 1997 Nonlinear magnetohydrodynamics (Cambridge University Press).

Browning, P.K. 1988 Phys. Rep. 196, 329

Freidberg, J.P. 1987 Ideal magnetohydrodynamics (Plenum, New York) 